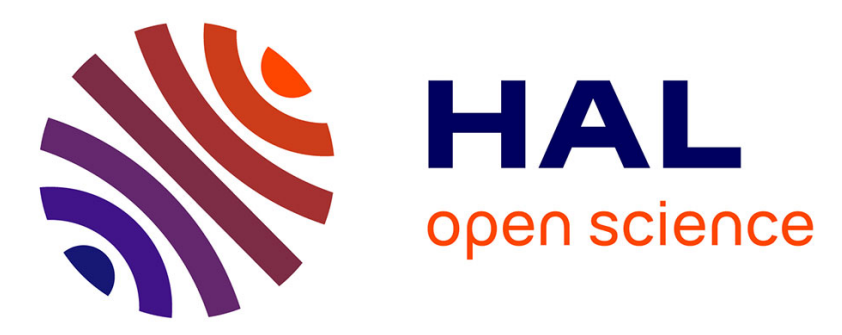

\title{
Wavelength selective light-induced magnetic effects in the binuclear spin crossover compound $[\mathrm{Fe}(\mathrm{bt})(\mathrm{NCS}) 2] 2(\mathrm{bpym})$
}

Nawell Ould-Moussa, Elzbieta Trzop, S. Mouri, Samir Zein, Gábor Molnár, Ana B. Gaspar, Eric Collet, Marylise Buron-Le Cointe, José Antonio Real, Sergei Borshch, et al.

\section{To cite this version:}

Nawell Ould-Moussa, Elzbieta Trzop, S. Mouri, Samir Zein, Gábor Molnár, et al.. Wavelength selective light-induced magnetic effects in the binuclear spin crossover compound [Fe(bt)(NCS)2]2(bpym). Physical Review B: Condensed Matter and Materials Physics (1998-2015), 2007, 75 (5), pp.054101. 10.1103/PhysRevB.75.054101 . hal-00906085

\section{HAL Id: hal-00906085 https://hal.science/hal-00906085}

Submitted on 21 Nov 2013

HAL is a multi-disciplinary open access archive for the deposit and dissemination of scientific research documents, whether they are published or not. The documents may come from teaching and research institutions in France or abroad, or from public or private research centers.
L'archive ouverte pluridisciplinaire HAL, est destinée au dépôt et à la diffusion de documents scientifiques de niveau recherche, publiés ou non, émanant des établissements d'enseignement et de recherche français ou étrangers, des laboratoires publics ou privés. 


\title{
Wavelength selective light-induced magnetic effects in the binuclear spin crossover compound $\left\{\left[\mathrm{Fe}(\mathbf{b t})(\mathrm{NCS})_{2}\right]_{2}(\mathrm{bpym})\right\}$
}

\author{
N. Ould Moussa, ${ }^{1,3}$ E. Trzop, ${ }^{2}$ S. Mouri, ${ }^{3}$ S. Zein, ${ }^{4}$ G. Molnár, ${ }^{1}$ A. B. Gaspar, ${ }^{5}$ E. Collet,${ }^{2, *}$ M. Buron-Le Cointe, ${ }^{2}$ \\ J. A. Real, ${ }^{5, \dagger}$ S. Borshch, ${ }^{4}$ K. Tanaka, ${ }^{3}$ H. Cailleau, ${ }^{2}$ and A. Bousseksou ${ }^{1, \$}$ \\ ${ }^{1}$ Laboratoire de Chimie de Coordination, CNRS UPR8241, 205 route de Narbonne, 31077 Toulouse, France \\ ${ }^{2}$ Groupe Matière Condensée et Matériaux, CNRS UMR6626, Campus Beaulieu, Université de Rennes 1, 35042 Rennes Cedex, France \\ ${ }^{3}$ Department of Physics, Graduate School of Science, Kyoto University, Kyoto 606-8502, Japan \\ ${ }^{4}$ Ecole Normale Supérieure de Lyon, 46, allée d'Italie, 69364 Cedex, Lyon, France \\ ${ }^{5}$ Institut de Ciència Molecular, Universitat de València, Edificio de Institutos de Paterna, P.B. Box 22085, València 46071, Spain
}

(Received 23 May 2006; published 1 February 2007)

\begin{abstract}
Using Fourier transform infrared spectroscopy, x-ray diffraction, and magnetic susceptibility measurements under light irradiation, the selective light-induced excited spin state trapping (LIESST) and the reversibleLIESST effect have been evidenced and studied in depth in the binuclear spin crossover compound $\{[\mathrm{Fe}(\mathrm{bt})$ $\left.\times(\mathrm{NCS})_{2}\right]_{2}$ bpym\}. In this system, each magnetic site can switch from low spin (LS) to high spin (HS), so that three states exist, namely, the LS-LS, HS-LS, and HS-HS. All these techniques shine a new light on the high phototunability of this system. In addition to the direct photoswitching from the LS-LS to the HS-LS or to the HS-HS state, here we show that photoinduced switching between the excited photoinduced states can be triggered in a reversible way: from HS-LS to HS-HS (irradiation around $800 \mathrm{~nm}$ ), or reverse from HS-HS to HS-LS (irradiation around $1300 \mathrm{~nm}$ ). The nature of the intermediate HS-LS state during the thermal and light-induced spin state changes is also discussed by comparing the spectroscopic measurements and the structural analysis. The loss of inversion symmetry in the HS-LS molecular state, where the two magnetic $\mathrm{Fe}$ sites are no more equivalent, is not accompanied by any long-range ordering of the noncentrosymmetric molecules in the crystal. Therefore the continuous double-step spin conversion corresponds to a double crossover.
\end{abstract}

DOI: 10.1103/PhysRevB.75.054101

PACS number(s): 75.30.Wx, 64.60.My, 78.30.-j

\section{INTRODUCTION}

The possibility of combining two properties like magnetic coupling and spin crossover (SCO) in a polynuclear molecule was realized by $\mathrm{O}$. Kahn almost two decades ago. ${ }^{1}$ It was foreseen that the magnetic signal of the material could be modulated as a result of the interplay between the two phenomena. The first stage in this direction was the synthesis of 2,2' -bipyrimidine (bpym) bridged iron(II) binuclear SCO compounds. A revision of this family of compounds has been recently reported. ${ }^{2}$ The most singular compound of this family is $\left\{\left[\mathrm{Fe}(\mathrm{bt})(\mathrm{NCS})_{2}\right]_{2}\right.$ bpym $\}$, represented in Fig. 1, where bt is the peripheral organic ligand 2,2' -bithiazoline, which represents the first example of thermally induced two-step spin conversion in a binuclear species. ${ }^{3}$

A singular feature stemming from the binuclear nature of this compound is the generation of a negative short-range interaction, which can be called as an antiferromagnetic-like interaction. $^{4,5}$ In contrast to molecular magnetism, such an interaction has essentially a steric and/or elastic origin. The very simple idea was that a volume change of one iron center of the dimetallic molecule due to the spin crossover phenomenon leads to an increase of the energy gap between the high spin (HS) and the low spin (LS) levels of the second iron metal. This effect arises from an intramolecular pressure generated by the first metallic center, leading to a shift of the crossover temperature of the second center toward higher temperatures, generating thus, a two-step spin crossover. In the same paper $^{3}$ a macroscopic theoretical approach using negative interaction of pairs (intramolecular) has been pro- posed, then later a microscopic Ising-like model has been introduced based on a ferro-like cooperative interaction for the long-range interactions and an antiferro-like interaction for the short-range interaction. ${ }^{4}$ Both interactions have been treated in the mean-field approximation. This model reproduced well the main two-step spin crossover behaviors. Later, the antiferro-like interaction was treated with an exact approach in Ref. 5.

It has been demonstrated in this work that for a certain balance between negative and positive interactions, a twostep spin crossover may be observed even for homogeneous ferro-like interaction in the material. ${ }^{5}$ Such a situation led to a double symmetry breaking (reentrant effect) around two Néel temperatures, leading to an antiferro-like phase sepa-

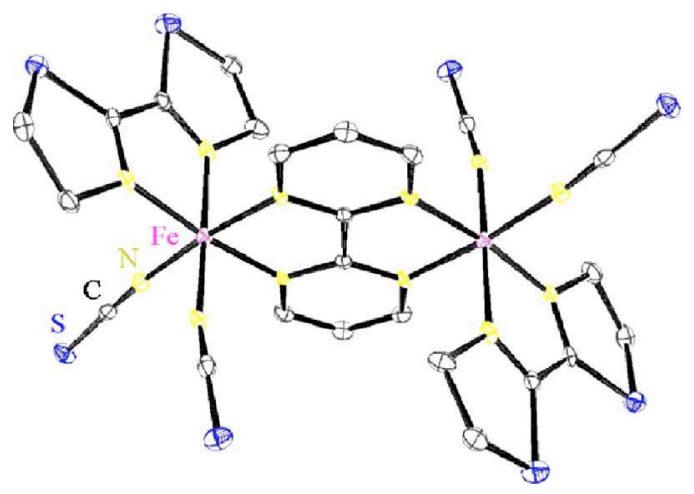

FIG. 1. (Color online) Structure of the binuclear molecule $\left\{\left[\mathrm{Fe}(\mathrm{bt})(\mathrm{NCS})_{2}\right]_{2}\right.$ bpym $\}$ located on inversion symmetry site. 
rated by two Néel temperatures. Boukheddaden et al. have also evidenced that negative short-range interactions may induce a sigmoïdal behavior in the relaxation curves after a photoswitching effect at liquid helium temperatures. ${ }^{6}$

The crystal structure of the complex $\left\{\left[\mathrm{Fe}(\mathrm{bt})(\mathrm{NCS})_{2}\right]_{2}\right.$ bpym $\}$, represented in Fig. 1, has been solved very recently at $293 \mathrm{~K}, 240 \mathrm{~K}, 175 \mathrm{~K}$, and $30 \mathrm{~K}$. At all these temperatures the crystal remains in the $P_{\overline{1}}$ space group. The structure consists of centrosymmetric binuclear units where each iron(II) atom is surrounded by two $\mathrm{NCS}^{-}$ anions in the cis position, two nitrogen atoms of the bridging bpym ligand, with the remaining positions occupied by the peripheral bt ligand. The iron atom is in a severely distorted octahedral $\left[\mathrm{FeN}_{6}\right]$ environment. The average $\mathrm{Fe}-\mathrm{N}$ bond length of 2.15(9) $\AA$ indicates that this compound is in the high-spin state ([HS-HS]) at $293 \mathrm{~K}$. Crystal structure determinations at $240 \mathrm{~K}, 175 \mathrm{~K}$, and at $30 \mathrm{~K}$ gave a comparable cell to that seen at $293 \mathrm{~K}$ but reduced in volume. At $30 \mathrm{~K}$, the average $\mathrm{Fe}-\mathrm{N}$ distance is $1.958(4) \AA$, showing that the structure is clearly low spin ([LS-LS]). At $175 \mathrm{~K}$, the Fe-N average bond length of 2.052(11) $\AA$ suggests the occurrence of an intermediate phase compatible with an intermediate state halfway between the high and low spin states. The fact that this state consists of [HS-LS] pairs can be inferred from Mössbauer spectroscopic studies of Ksenofontov et al. under applied external magnetic field. ${ }^{8,9}$

Density functional theory (DFT) calculations on the binuclear complex $\left\{\left[\mathrm{Fe}(\mathrm{bt})(\mathrm{NCS})_{2}\right]_{2}\right.$ bpym $\}$ have also been performed with the goal to characterize energies, geometric, and electronic structure in three possible electronic states [LSLS], [HS-LS], and [HS-HS]. ${ }^{10}$ The self-consisted procedure converged to three states with spin densities corresponding to expected values of local spins: $0-0,0-2$, and 2-2. If the first and the last states have been obtained, supposing a center of the symmetry in the complex, the mixed state is clearly characterized by loss of the central symmetry. However, in all states the bridging ligand keeps its quasiplanar structure. The iron-nitrogen distances vary from 2.00 to $2.29 \AA$ for the high-spin center, whereas for the low-spin center the dispersion is much smaller: $1.94-1.99 \AA$. The mean iron-nitrogen distance $(2.06 \AA)$ is slightly lower than halfway between the [LS-LS] and [HS-HS] states (1.97 and $2.17 \AA$, respectively). The energy of the mixed state lies also slightly lower than halfway between the [LS-LS] and [HS-HS] states, thus satisfying a necessary condition ${ }^{3}$ for the two-step character of the spin transition. In the language of phenomenological models, this stabilization of the mixed state can be seen as a manifestation of the "antiferro"-like elastic interaction between individual centers. Quantum-chemical calculations with the broken symmetry method also allowed determination of the value of the electronic antiferromagnetic exchange interaction, which is active only in the hightemperature [HS-HS] state.

Following the first photomagnetic studies by Létard et al. ${ }^{11-13}$ who observed a LS-LS $\rightarrow$ HS-HS photoconversion under red light irradiation, the photomagnetic properties of the $\left\{\left[\mathrm{Fe}(\mathrm{bt})(\mathrm{NCS})_{2}\right]_{2}\right.$ bpym $\}$ compound have been reexamined. ${ }^{14}$ Using Raman spectroscopy, it was possible to give spectroscopic evidence for the LS-LS $\rightarrow$ HS-HS photo- switching process with excitation in the red region. Moreover, we have shown that infrared light $(1342 \mathrm{~nm})$ selectively photoconverts the fundamental LS-LS state to the HS-LS state, revealing a direct experimental evidence of the symmetry breaking in this compound, as predicted in 1993, ${ }^{5}$ and in agreement with Mössbauer spectroscopy experiments. $^{2}$

In the present work, we investigate in-depth the lightinduced excited spin state trapping (LIESST) phenomenon in the compound $\left\{\left[\mathrm{Fe}(\mathrm{bt})(\mathrm{NCS})_{2}\right]_{2} \mathrm{bpym}\right\}$ by using different and complementary techniques [Fourier transform infrared (FTIR), x-ray diffraction, and magnetic susceptibility measurements] and DFT calculations. The results presented here make it possible to address two questions of primary importance: is there any long-range ordering in the crystal accompanying the loss of inversion symmetry of the HS-LS molecular state? Is it possible to trigger photoinduced effects between excited states and what is their nature?

\section{EXPERIMENTS}

Photomagnetic measurements were performed on a thin layer of polycrystalline compound (synthesis according to Ref. 3) using a magnetic property measurement system (Quantum Design) operated at $1 \mathrm{~T}$ between $2 \mathrm{~K}$ and $400 \mathrm{~K}$ and equipped with a sample rod allowing irradiation of the sample via fiber optics. For the irradiation setup two lasers were used: a Nd: $\mathrm{YVO}_{4}$ laser $\left(1342 \mathrm{~nm}, 10 \mathrm{~mW} / \mathrm{cm}^{2}\right)$ and a laser diode $\left(785 \mathrm{~nm}, 20 \mathrm{~mW} / \mathrm{cm}^{2}\right)$. Infrared absorption spectra of $\left\{\left[\mathrm{Fe}(\mathrm{bt})(\mathrm{NCS})_{2}\right]_{2}\right.$ bpym $\}$ were acquired in $\mathrm{KBr}$ pellets by means of a Shimadzu Prestige-21 FTIR spectrometer. The sample was pelletized without grinding. Spectra were recorded between room temperature and $15 \mathrm{~K}$ in the $4000-400 \mathrm{~cm}^{-1}$ frequency range. The experimental setup allowed us to irradiate the sample with red $(785 \mathrm{~nm})$ or nearinfrared $(1342 \mathrm{~nm})$ light at $15 \mathrm{~K}$ and record IR spectra of the irradiated zone. The light of the HeNe laser (calibrating the interferometer in the FTIR instrument) was obstructed by a needle. UV visible (UV-VIS) absorption spectra of $\left\{\left[\mathrm{Fe}(\mathrm{bt})(\mathrm{NCS} 2)_{2}\right]_{2}(\right.$ bpym $\left.)\right\}$ were obtained in $\mathrm{KBr}$ pellets using a Shimadzu UV-3150 spectrometer. Spectra were recorded between room temperature and $15 \mathrm{~K}$ in the $300-1500 \mathrm{~nm}$ wavelength range. Thermal and photoinduced switching were investigated by $x$-ray diffraction on single crystals, obtained by cutting of twined ones as received from the synthesis. X-ray data were collected on a four-circle Oxford Diffraction Xcalibur 3 diffractometer (Mo $K \alpha$ radiation) with a two-dimensional (2D) sapphire 3 charge coupled device (CCD) detector, on samples with typical sizes around $100 \times 100 \times 100 \mu \mathrm{m}^{3}$ in different experimental conditions. The single crystals were mounted either in an Oxford Diffraction Helijet helium-flow cryostat, allowing temperatures reaching $20 \mathrm{~K}$, or in an Oxford Cryosystems nitrogen-flow cryostat, allowing a better control of the temperature down to $78 \mathrm{~K}$. The unit cell parameters and the data reduction were obtained with CRYSALIS software from Oxford Diffraction. ${ }^{15}$ The structures were solved with SIR- $97^{16}$ and refined with SHELXL97. ${ }^{17}$ Typical results of the structure refinement of the stable and photoinduced states gave final $\mathrm{R}$ factor 0.03 
$<\mathrm{R}<0.055$. The detailed structural analysis will be published elsewhere. ${ }^{18}$

\section{DENSITY FUNCTIONAL CALCULATIONS}

The vibrational frequencies of the complex $\left\{\left[\mathrm{Fe}(\mathrm{bt})(\mathrm{NCS} 2)_{2}\right]_{2}(\mathrm{bpym})\right\}$ in three spin states have been calculated starting from equilibrium geometries obtained in previous DFT calculations. ${ }^{10}$ As it is meaningless to compute frequencies with a method other than that used for the stationary points determination, the computation details are exactly the same. The frequency calculations have been performed using GAUSSIAN 98 (A7 version) package. ${ }^{19}$ The LANL2DZ basis set, including the double- $\zeta$ basis with the Los Alamos effective core potential for $\mathrm{Fe}, \mathrm{S}$, and $\mathrm{Se}$, and the Dunning-Huzinaga all-electron double- $\zeta$ basis set with polarization functions for the $\mathrm{H}, \mathrm{C}$, and $\mathrm{N}$ atoms, have been used. $^{20,21}$ The B3LYP* exchange correlation functional, specially adapted for spin transition systems, has been chosen. ${ }^{22}$ Special attention has to be paid to the assignment of calculated vibrational frequencies and their comparison in different spin states. As the shape of force fields changes with the spin state, not only frequencies of normal vibrations vary but also their composition. The comparison of different normal vibrations based on their visual representation, as it has been done in several publications, can be inexact if we do not deal with characteristic vibrations. In order to avoid this difficulty, we proposed a method based on the calculation of projections of normal vibration vectors in one spin state on vectors in another state and then on the selection of counterparts corresponding to the most important projection coefficients.

\section{RESULTS AND DISCUSSION}

\section{A. Magnetic and spectroscopic studies}

Figures 2(a) and 2(b) show the variation of the $\chi_{m} T$ product (where $\chi_{m}$ stands for the molar magnetic susceptibility) upon light irradiation at $10 \mathrm{~K}$ and subsequent heating as a function of the time and temperature, respectively. The sample was first cooled to $10 \mathrm{~K}$ in the dark to obtain the LS-LS state $\left(\chi_{m} T=0.3 \mathrm{~cm}^{3} \mathrm{~mol}^{-1} \mathrm{~K}\right)$. It was then irradiated by red light $(t=0 \mathrm{~min})$, leading to an increase of the $\chi_{m} T$ product to ca. $1.4 \mathrm{~cm}^{3} \mathrm{~mol}^{-1} \mathrm{~K}$. After saturation of the magnetization $(t=610 \mathrm{~min})$, we heated the sample to $45 \mathrm{~K}$. Upon heating, the magnetization increased strongly and at $45 \mathrm{~K}$ the $\chi_{m} T$ product reached a value of $4.4 \mathrm{~cm}^{3} \mathrm{~mol}^{-1} \mathrm{~K}$. As it was shown earlier, this behavior can be associated with the formation of HS-HS pairs (LIESST effect), in which the magnetic moment is quenched at low temperatures due to the antiferromagnetic coupling between the two HS iron ions. ${ }^{11}$ In a next step, the sample (still in the HS-HS state!) was cooled back to $10 \mathrm{~K}(t=660 \mathrm{~min})$ and was irradiated by IR light $(t=920 \mathrm{~min})$. This irradiation led only to a slight increase of the $\chi_{m} T$ product from 1.4 to $1.9 \mathrm{~cm}^{3} \mathrm{~mol}^{-1} \mathrm{~K}$. Subsequent heating to $45 \mathrm{~K}(t=1410 \mathrm{~min})$ led to a $\chi_{m} T$ value of $3.2 \mathrm{~cm}^{3} \mathrm{~mol}^{-1} \mathrm{~K}$, which is significantly lower than the value of $4.4 \mathrm{~cm}^{3} \mathrm{~mol}^{-1} \mathrm{~K}$ obtained after red light irradiation. We can explain tentatively this change in the magnetic behavior by a reverse LIESST effect, i.e., the infrared light
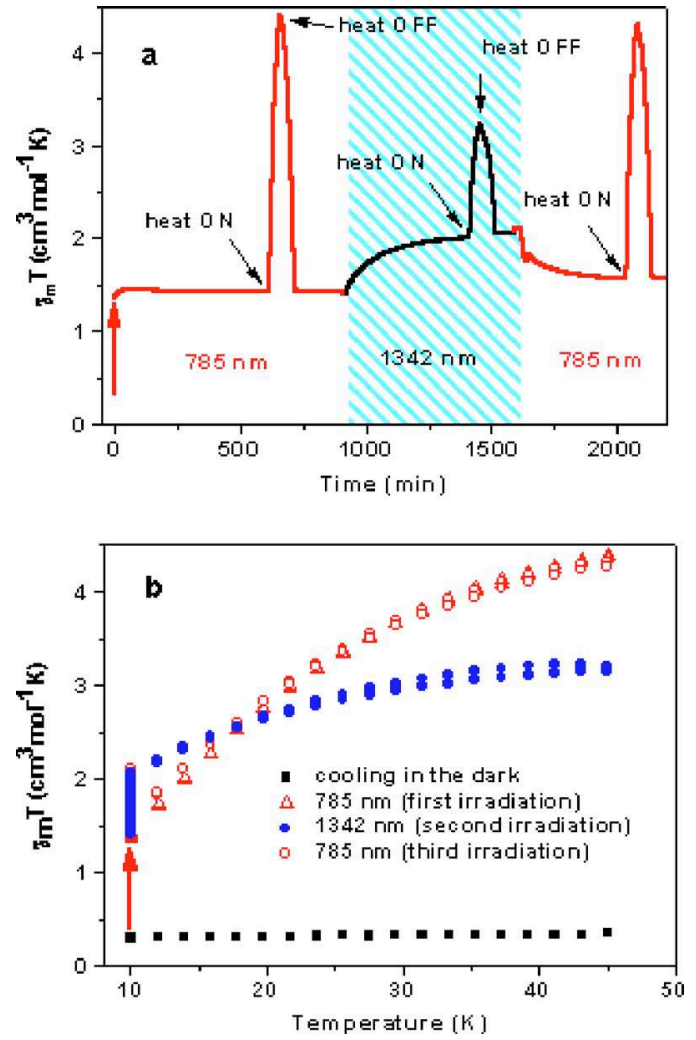

FIG. 2. (Color online) Evolution of the $\chi_{m} T$ product in $\left\{\left[\mathrm{Fe}(\mathrm{bt})(\mathrm{NCS})_{2}\right]_{2}\right.$ bpym $\}$ upon successive red, infrared, and red light irradiations as a function of time (a) and temperature (b). The irradiation of the sample was carried out at $10 \mathrm{~K}$ in each case. When the magnetization has reached a constant value, the sample was heated up to $45 \mathrm{~K}$ and cooled back to $10 \mathrm{~K}$.

transforms (at least partially) the HS-HS molecules into HS-LS pairs. As a final step, we cooled back the sample to $10 \mathrm{~K}(t=1455 \mathrm{~min})$ and irradiated it again by red light $(t=1600 \mathrm{~min})$, leading to a slight decrease of the magnetization. When heating to $45 \mathrm{~K}(t=2030 \mathrm{~min})$, we observed again the strong increase of the $\chi_{m} T$ product to $4.4 \mathrm{~cm}^{3} \mathrm{~mol}^{-1} \mathrm{~K}$.

These photomagnetic experiments suggest that irradiating the HS-HS state by infrared light leads to the formation of the HS-LS state and subsequent red light irradiation of the HS-LS state leads again to the pure HS-HS form. To clarify these hypotheses we decided to employ FTIR spectroscopy. (Raman spectroscopy cannot be readily used for such double-excitation experiments because of the difficulties to probe the same volume by red and infrared lights in the strongly absorbing powder sample. On the other hand, in infrared spectroscopy the dilution of the sample in $\mathrm{KBr}$ pellets allows good control of the irradiation volume.) Figure 3 displays IR absorption of the sample in the region of $\mathrm{CN}$ stretching modes at different temperatures and under light irradiation.

At $280 \mathrm{~K}$ one can observe two $\mathrm{CN}$ stretching modes at around 2080 and $2090 \mathrm{~cm}^{-1}$. These modes were assigned to the HS state. ${ }^{13}$ In the plateau region $(180 \mathrm{~K})$, two new modes appear at around 2110 and $2125 \mathrm{~cm}^{-1}$, which can be associated with the LS state. ${ }^{13}$ These modes increase further in 

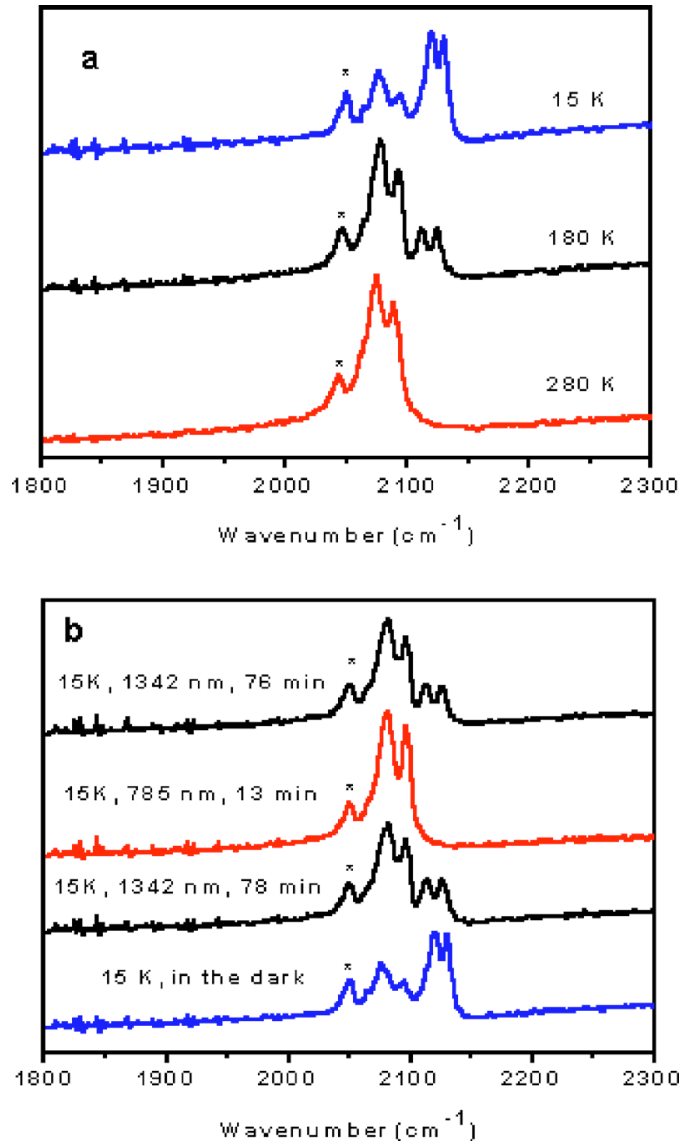

FIG. 3. (Color online) (a) Infrared absorption spectra of $\left\{\left[\mathrm{Fe}(\mathrm{bt})(\mathrm{NCS})_{2}\right]_{2}\right.$ bpym $\}$ at different temperatures and (b) upon successive infrared, red, and infrared light irradiations at $15 \mathrm{~K}$. ( ${ }^{*}$ The signal around $2050 \mathrm{~cm}^{-1}$ is an artifact of the spectrometer.)

intensity when cooling to $100 \mathrm{~K}$ and in the same time the intensity of the HS modes at 2080 and $2090 \mathrm{~cm}^{-1}$ decreases. There remains a small amount of residual HS fraction even at $15 \mathrm{~K}$, but this has also been observed in Mössbauer experiments previously. ${ }^{3}$ When the sample is shined with $1342 \mathrm{~nm}$ light at $15 \mathrm{~K}$ the intensity of HS modes increases for the detriment of LS modes and the spectrum of the photoinduced state corresponds to the spectrum in the plateau region $(180 \mathrm{~K})$. In the next step, the sample was irradiated at $15 \mathrm{~K}$ by red light, leading to a pure HS spectrum (HS-HS state). Then, irradiation of this HS-HS state by IR light led again to the HS-LS spectrum. These experiments corroborate perfectly the photomagnetic measurements and give therefore a spectroscopic evidence for the following photoinduced effects:

$$
\mathrm{LS}_{1342} \underset{\mathrm{nm}}{\mathrm{HS}-\mathrm{LS}} \underset{1342 \mathrm{~nm}}{\rightleftarrows} \mathrm{HS}-\mathrm{HS}
$$

In other words, the nature of the photoinduced state only depends on the irradiation wavelength and not on the initial state (stable or photogenerated). An open question is the origin of this effect. The most obvious explanation would be to suppose that it is simply related to the absorbance of the sample at $1342 \mathrm{~nm}$ in the three states. Therefore we have

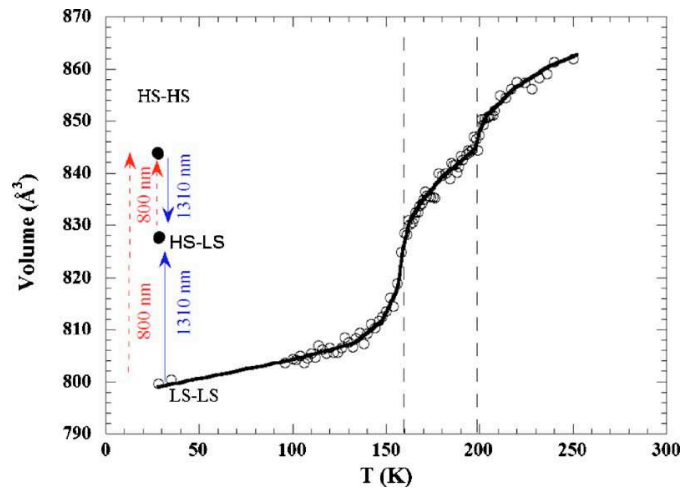

FIG. 4. (Color online) Temperature dependence of the unit cell volume (where no hysteresis is observed) and effect of $1310 \mathrm{~nm}$ and $808 \mathrm{~nm}$ laser irradiations at $23 \mathrm{~K}$.

recorded UV-VIS spectra of the sample in the three states (LS-LS, HS-LS, HS-HS). These spectra confirmed that at $1342 \mathrm{~nm}$ the sample has the highest absorption in the LS-LS state, but no significant difference was observed between the plateau region (HS-LS) and room temperature (HS-HS) (see supplementary material ${ }^{23}$ ). Time-resolved experiments will therefore be necessary to better understand the dynamics in the relaxation process that follows light excitations at different wavelengths.

\section{B. Structural analysis of the photo-switching}

In the family of spin transition materials, it is well known that important structural changes occur at the level of the coordination sphere surrounding $\mathrm{Fe}$ atoms, associated with the change of electronic state. A typical signature is the shift of the Fe-N distances from around $2.2 \AA$ in the HS state to $2.0 \AA$ in the LS state and thus important changes in the volume of the crystal take place. ${ }^{24}$

Figure 4 shows the temperature dependence of the volume, where two anomalies at around $200 \mathrm{~K}$ and $160 \mathrm{~K}$ sign the presence of the intermediate HS-LS state. At $23 \mathrm{~K}$, the crystal in the LS-LS state switches by the irradiation at $808 \mathrm{~nm}\left(0.26 \mathrm{~mW} / \mathrm{cm}^{2}\right)$ to the HS-HS state, characterized by a volume of ca. $844 \AA^{3}$, whereas the irradiation at $1310 \mathrm{~nm}\left(0.13 \mathrm{~mW} / \mathrm{cm}^{2}\right)$ switches the crystal to the HS-LS state, characterized by a volume of ca. $828 \AA^{3}$. The complete transformations take place within a few minutes, as shown in Fig. 5(a). In agreement with the magnetic and FTIR data, it is possible to switch in a reversible way between the two photoinduced states (HS-HS and HS-LS) by choosing the appropriate laser excitation wavelength. Indeed, the HS-HS state is generated from both the LS-LS and HS-LS states by irradiation at $808 \mathrm{~nm}$, and the HS-LS state is generated from the LS-LS and HS-HS states by irradiation at $1310 \mathrm{~nm}$.

These results show that one can easily increase and decrease the volume of the crystal by laser excitation. It is noticed that the change of the volume occurs gradually with a gradual shift of the Bragg peaks in the reciprocal space (see the movie in supplementary material ${ }^{23}$ ), contrary to what is observed in the case of orthorhombic $\left[\mathrm{Fe}(\mathrm{PM}-\mathrm{BiA})_{2}(\mathrm{NCS})_{2}\right]$ (Ref. 25) or $\left[\mathrm{Fe}\left(2\right.\right.$-picolylamine) $\left.{ }_{3}\right] \mathrm{Cl}_{2} \mathrm{EtOH},{ }^{26}$ where a phase 

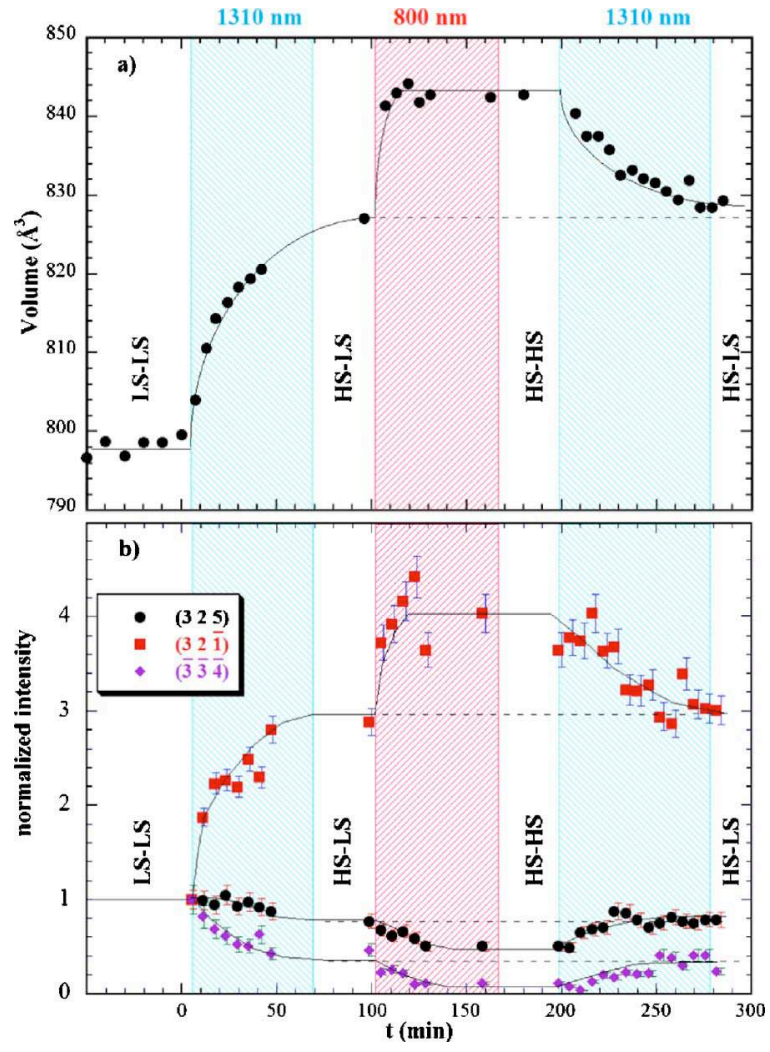

FIG. 5. (Color online) Time dependence of the volume of the unit cell (a) and intensity of some Bragg peaks (b) under laser irradiation at $1310 \mathrm{~nm}$ and $808 \mathrm{~nm}$ at $23 \mathrm{~K}$.

separation process takes place, indicating that the cooperative interactions between the photoinduced molecules are not so strong and the concentration of photodoped molecules is homogeneously increased within the crystal. In addition to the change of the volume, the change of the intensity of some Bragg peaks is a strong signature: the structural reorganization affects the intensity of the Bragg reflections. Figure 5(b) shows the time dependence of the intensity of some Bragg peaks at $23 \mathrm{~K}$, between the LS-LS, HS-LS, and HS-HS states.

Both Figs. 5(a) and 5(b) demonstrate the reversible structural rearrangement between the different states. Furthermore, the structures obtained for the photoinduced HS-LS and HS-HS states ${ }^{18}$ are comparable with the adequate ones at thermal equilibrium, ${ }^{7}$ showing that the photoswitching is complete.

Indeed, more detailed information was obtained at the molecular level by solving the structure of the different states (23 K for the photoinduced HS-HS and HS-LS states, $293 \mathrm{~K}$ and $250 \mathrm{~K}$ for the HS-HS state, $189 \mathrm{~K}, 185 \mathrm{~K}$ and $180 \mathrm{~K}$ and $175 \mathrm{~K}$ for the HS-LS state, at 130 and $23 \mathrm{~K}$ for the LS-LS state).

As already performed for the different states at thermal equilibrium, ${ }^{7}$ the different structures and especially the ones of the photoinduced HS-HS and HS-LS states were refined in the $P_{\overline{1}}$ space group, where the binuclear molecules are located on an inversion symmetry site (Fig. 1). The main signature is associated with the important changes of the $\langle\mathrm{Fe}-\mathrm{N}\rangle$ bond length from 1.954(3) $\AA$ in the LS-LS state to
2.081(5) $\AA$ in the photoinduced HS-LS state and 2.152(3) $\AA$ in the photoinduced HS-HS state. These values are very similar to the ones observed during the thermal transition on the plateau in the HS-LS state (that is in the range of 2.05-2.09 $\AA$ between $175 \mathrm{~K}$ and $189 \mathrm{~K}$ ) and in the HS-HS state $2.150(6)-2.160(6) \AA$ between $250 \mathrm{~K}$ and $300 \mathrm{~K}$. Therefore, there is a clear structural signature associated with the generation of the HS-HS or HS-LS states, and here again it is reversible. This result is very interesting in that it demonstrates the possibility to generate photoinduced transformation between excited states (photoinduced HS-HS and HS-LS states) and it opens fascinating possibilities both from the experimental, theoretical, and applied point of view.

\section{Symmetry of the HS-LS state}

As it is indicated below and demonstrated in Ref. 14, the formation of photoinduced HS-LS state involves spontaneous symmetry breaking. To be exact, for binuclear spincrossover compounds, it is a double symmetry breaking at two Néel temperatures, when the HS population of one metallic center becomes different than the HS population of the second center in the network. In other words, if we defined $n=n_{\mathrm{HSA}}-n_{\mathrm{HSB}}$, where $\mathrm{A}$ and $\mathrm{B}$ design the two metallic centers of the molecule, $n$ has the definition of an order parameter in the general Landau theory and the symmetry breaking is obtained in the temperature interval between $T_{\mathrm{N}}^{+}$and $T_{\mathrm{N}}^{-}$for which $n$ becomes $\neq 0 .^{5}$ From the structural point of view, it is essential to be more explicit on the structural symmetry changes at $T_{\mathrm{N}}^{+}$and $T_{\mathrm{N}}^{-}$, i.e., space group and molecular site symmetry changes, and eventually to indicate which type of structural symmetry breaking may be observed.

The symmetry breaking in the HS-LS state is an important issue that has to be discussed. Raman and Mössbauer spectroscopy $8,9,14$ have evidenced that the intermediate HS-LS state observed under irradiation at $1342 \mathrm{~nm}$ or in the temperature range of $160-200 \mathrm{~K}$ is not a mixture of HS-HS and LS-LS states but that a third molecular state appears. This HS-LS state is associated with the loss of the inversion symmetry on the molecule. ${ }^{8,9,14}$ In order to describe the crystalline nature of this HS-LS state, one has to describe how the HS-LS molecules rearrange in the crystal and whether or not the symmetry breaking at the molecular level appears on the three-dimensional (3D) crystalline structure. A comparison of these experimental results with x-ray diffraction data makes it possible to discuss it in terms of structural broken symmetry. The structural analysis of the HS-HS and LS-LS spin states of the binuclear compounds describe them as $P_{\bar{l}}$. The molecules are located on inversion symmetry sites, so that the two Fe sites of the molecules are equivalent by symmetry, as schematically shown in Fig. 6(a). In the HS-LS state, different possibilities exist regarding the 3D crystalline order, since when binuclear molecules in the HS-LS state form, the inversion symmetry is lost on the molecule. From the space group point of view, it means that another symmetry operator may have to be lost. The Landau theory of phase transition and ordering phenomena was applied to the spin transition, discussing the possibility of ordering between HS and LS sites. ${ }^{27,28}$ Therefore the crystalline structure of the 

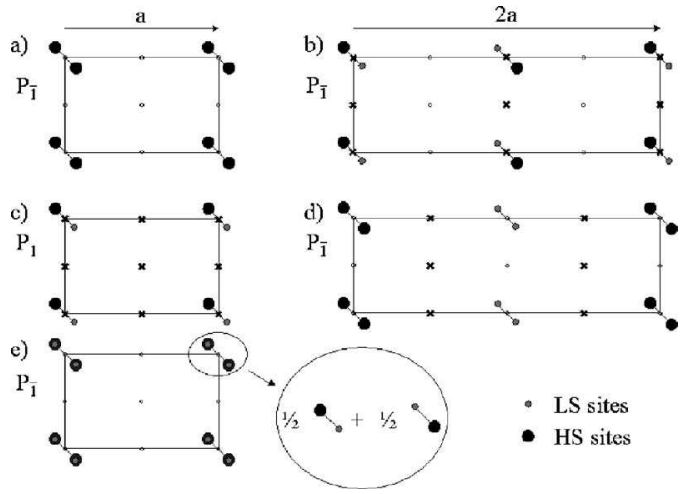

FIG. 6. Schematic crystalline structures where the molecules are located on inversion symmetry site. Crosses schematically indicate the lost inversion symmetry operators.

binuclear complex may be discussed as a set of mononuclear sites related by inversion symmetry, where the population of HS state may differ from site-to-site.

One possibility is the loss of translation symmetry [Fig. 6(b)], where the loss of inversion symmetry couples with a cell doubling along one direction, for example. The inversion symmetry between the molecules is kept so that the space group remains $P_{\bar{l}}$. In such a case, superstructure reflections should be observed since the structural rearrangement at the molecular level between HS and LS sites is important, as observed in $\left[\mathrm{Fe}(2 \text {-picolylamine })_{3}\right] \mathrm{Cl}_{2} \mathrm{EtOH},{ }^{26-28}$ or in some other compounds ${ }^{29,30}$ as well. We looked carefully for such signatures in the diffraction data, but no change of translation symmetry was observed.

Another possibility is that the translation symmetry is kept and that the inversion symmetry on the molecules and between the molecules is lost [Fig. 6(c)]. In such a case, the space group changes from $P_{\bar{l}}$ to $P_{1}$. The atomic motions between the HS and LS states are so large that when the structure of the intermediate state is solved, significant differences between Fe-N bond lengths of the HS and LS sites should be observed. We solved the structure in the $P_{1}$ space group and such differences could not be observed. It also has to be noticed that in such a case, the HS-LS state will be associated with two symmetry-breaking order parameters (one describing the loss of inversion symmetry on the molecules and the other one between the molecules). As it is well known from the Landau theory of phase transition, when two such symmetry-breaking order parameters couple in the development of the free energy in a biquadratic term, only first-order phase transition will be possible between the HS-HS and HS-LS states and between the HS-LS and LS-LS spin states. In this compound, a gradual change occurs between the HS-HS and HS-LS states and also between the HS-LS and LS-LS states. Therefore, by considering the Landau theory, it is another indication that the HS-LS state cannot be $P_{1}$, since two first-order transitions should take place. The case where an ordering of binuclear molecules in the HS-HS state and binuclear molecules in the LS-LS state takes place [Fig. 6(d)] will also be associated with a change of the translation symmetry not observed here, as mentioned before. In addition, Mössbauer spectroscopy and Raman spectroscopy indicated that the symmetry is broken at the molecular level. Therefore, from the x-ray diffraction experiments, it comes out that the space group remains $P_{\bar{l}}$ and that no change of symmetry occurs on the average $3 \mathrm{D}$ structure. In comparison with the Raman scattering experiments, the results reveal that this intermediate state is made of HS-LS binuclear molecules, which are fluctuating in space and eventually in time. As Raman scattering is a fast and local probe, it can watch the symmetry breaking on the molecule. However, as no correlation exists from site-to-site, x-ray diffraction will only see an average state where molecules will have a probability of $\frac{1}{2}$ to be in the HS-LS molecular state and the same probability to be in the HS-LS one [Fig. 6(e)]. The disordered structure $P_{l}$ of the HS-LS crystalline state is then isostructural to both the HS-HS and LS-LS ones.

The value of the $\langle\mathrm{Fe}-\mathrm{N}\rangle$ bond length in the HS-LS state on the plateau or in the photoinduced state varies between $2.05 \AA(175 \mathrm{~K})$ and $2.09 \AA(189 \mathrm{~K})$. It is close to the average value of $2.05 \AA$ between the HS-HS $(\approx 2.15 \AA)$ and LS-LS states $(\approx 1.95(1) \AA)$ [Figs. 6(e) and 6(c)] that we should observe, if we assume that for the molecules in the HS-LS state the average value of the $\langle\mathrm{Fe}-\mathrm{N}\rangle$ bond length on the LS site is the same as for the completely LS-LS, and vice versa for the HS site. As pointed out before, a slight deviation from the average can be explained by the antiferro-like coupling between the two sites that will result in a local pressure.

\section{Density functional results}

It should be noted that these calculations are done for an isolated molecule. To be exact, it should be extended (more difficult) to the crystal. The calculated vibrational frequencies are given in the supplementary material. ${ }^{23}$ In the centrally symmetrical [LS-LS] and [HS-HS] states all normal vibrations can be divided in even and odd combinations. The frequency splitting between even and odd counterparts measures the elastic interaction strength between two vibrations localized in two parts of the molecule and connected by the inversion symmetry. The analysis of the shape of normal vibrations allows assigning the vibrations in the range $1420-1550 \mathrm{~cm}^{-1}$ to ring deformations and interring stretching of the bridging bpym ligand, in agreement with our previous assignments. ${ }^{14}$ The four frequencies around 2050-2100 $\mathrm{cm}^{-1}$ correspond to $\mathrm{CN}$ stretching vibrations, in agreement with our Raman and FTIR results. The even and odd vibrations are formed by "symmetric" and "antisymmetric" combination of local vibrations of two NCS ligands around each iron center. It can be seen that the splitting between even and odd components is rather small in the [LS-LS] and [HS-HS] states. In principle, two peaks could be expected for both states. Although the calculated frequencies (2046 and $2063 \mathrm{~cm}^{-1}$ for the [HS-HS] and 2093 and $2107 \mathrm{~cm}^{-1}$ for the [LS-LS] state) are slightly lower than the experimental ones (2080 and $2090 \mathrm{~cm}^{-1}$ for the [HS-HS], 2110 and $2125 \mathrm{~cm}^{-1}$ for the [LS-LS] states), the splitting between local "symmetric" and "antisymmetric" vibrations $\left(\sim 10-15 \mathrm{~cm}^{-1}\right)$ is nicely reproduced by the calculations. In the mixed [HS-LS] state the normal vibrations lose the sig- 
nature of even or odd representation and correspond to combinations of stretching vibrations around each iron center. The obtained four frequencies cover the whole range characteristic for both [LS-LS] and [HS-HS] states. This dispersion of vibrations of the same type also displays the loss of the dimer symmetry in the intermediate state.

\section{CONCLUSIONS}

In our previous paper ${ }^{14}$ we have demonstrated a selective photoswitching phenomena from the fundamental LS-LS state to the metastable HS-LS $(1342 \mathrm{~nm})$ and HS-HS $(647 \mathrm{~nm})$ states at low temperatures. In the present paper we provide clear experimental evidences from different techniques that a reversible photoconversion occurs between the two metastable states: HS-LS $\rightarrow$ HS-HS (red light irradiation) and the reverse HS-HS $\rightarrow$ HS-LS (infrared light). It appears, therefore, that the mechanism of selective photoexcitation is related to the reverse-LIESST phenomenon under infrared excitation. These results demonstrate the high phototunability of this system, associated with the selective and reversible photoswitching between the metastable states. Finally, it comes out that the photoinduced state only depends on the irradiation wavelength and not on the initial state.

From the x-ray analysis, it comes out that the HS-LS state is a mixture of molecules in the indistinguishable HS-LS and
LS-HS states. The gradual change of the volume during laser excitation or as a function of temperature characterizes a double-crossover phenomenon in a three-state model (LSLS, HS-LS, and HS-HS), in agreement with the magnetic properties, since no long-range structural symmetry breaking appears in the HS-LS state, neither at thermal equilibrium nor under laser irradiation. Therefore, in the model developed by Bousseksou et al., ${ }^{5}$ the order parameter (pure magnetic parameter) defining the difference of population on one site of HS and LS molecules, showing a symmetry breaking, concerns only the magnetic states and the local molecular structure (as inferred from the DFT calculations and vibrational spectra). It corresponds, from a structural point of view, with regard to the Landau theory of phase transitions, ${ }^{28}$ to the isostructural scenario.

\section{ACKNOWLEDGMENTS}

The authors are grateful to the JSPS, Japanese Office, for the grant to N. Ould Moussa supporting a part of her Ph.D. period in Japan at Kyoto University. Grants from the European FLASH network (MRTN-CT-2003-503641), NOE MAGMANet (515767-2), ANR "Fast-switch" (NT053_45333), Région Bretagne, the Spanish Ministerio de Educación y Ciencia (MEC) (CTQ 2004-03456/BQU), and the Spanish MEC for a research contract (Programa Ramón y Cajal) are also acknowledged.
*Corresponding author. Electronic address: eric.collet@univrennes1.fr

†Corresponding author. Electronic address: jose.a.real@uv.es

ॠCorresponding author. Electronic address: boussek@lcctoulouse.fr

${ }^{1}$ J. A. Real, J. Zarembowitch, O. Kahn, and X. Solans, Inorg. Chem. 26, 2939 (1987).

${ }^{2}$ J. A. Real, A. B. Gaspar, M. C. Muñoz, P. Gütlich, V. Ksenofontov, and H. Spiering, Top. Curr. Chem. 233, 49 (2004).

${ }^{3}$ J. A. Real, H. Bolvin, A. Bousseksou, A. Dworkin, O. Kahn, F. Varret, and J. Zarembowitch, J. Am. Chem. Soc. 114, 4650 (1992).

${ }^{4}$ A. Bousseksou, J. Nasser, J. Linares, K. Boukheddaden, and F. Varret, J. Phys. I 2, 1381 (1992).

${ }^{5}$ A. Bousseksou, F. Varret, and J. Nasser, J. Phys. I 3, 1463 (1993).

${ }^{6}$ K. Boukheddaden, I. Shteto, B. Hoo, and F. Varret, Phys. Rev. B 62, 14796 (2000).

${ }^{7}$ A. B. Gaspar, V. Ksenofontov, S. Reiman, P. Gütlich, A. L. Thompson, A. Goeta, M. Carmen Munoz, and J. A. Real, Chem.-Eur. J. 12, 9289 (2006); DOI: 10.1002/chem.200600559.

${ }^{8}$ V. Ksenofontov, H. Spiering, S. Reiman, Y. Garcia, A. B. Gaspar, N. Moliner, J. A. Real, and P. Gütlich, Chem. Phys. Lett. 348, 381 (2001).

${ }^{9}$ V. Ksenofontov, A. B. Gaspar, V. Niel, S. Reiman, J. A. Real, and P. Gütlich, Chem.-Eur. J. 10, 1291 (2004).

${ }^{10}$ S. Zein and S. A. Borshch, J. Am. Chem. Soc. 127, 16197 (2005).

${ }^{11}$ J.-F. Létard, J. A. Real, N. Moliner, A. B. Gaspar, L. Capes, O. Cador, and O. Kahn, J. Am. Chem. Soc. 121, 10630 (1999).
${ }^{12}$ G. Chastanet, A. B. Gaspar, J. A. Real, and J.-F. Létard, Chem. Commun. (Cambridge) 2001, 819 (2001).

${ }^{13}$ G. Chastanet, C. Carbonera, C. Mingotaud, and J.-F. Létard, J. Mater. Chem. 14, 3516 (2004).

${ }^{14}$ N. O. Moussa, G. Molnár, S. Bonhommeau, A. Zwick, S. Mouri, K. Tanaka, J. A. Real, and A. Bousseksou, Phys. Rev. Lett. 94, 107205 (2005).

${ }^{15}$ CRYSALIS RED, Oxford Diffraction, Ltd., vers. 1.171.26pre2 beta (2004).

${ }^{16}$ A. Altomare, M. C. Burla, M. Camalli, G. Cascarano, C. Giacovazzo, A. Guagliardi, A. G. G. Moliterni, G. Polidori, and R. Spagna., J. Appl. Crystallogr. 32, 115 (1999).

${ }^{17}$ G. M. Sheldrick, SHELX97, Program for the Refinement of Crystal Structures, University of Göttingen, Germany (1997).

${ }^{18}$ E. Trzop, M. Buron-Le Cointe, H. Cailleau, L. Toupet, G. Molnar, A. Bousseksou, A. B. Gaspar, J. A. Real, and E. Collet, J. Appl. Crystallogr. 40, 158 (2007).

${ }^{19}$ M. J. Frisch et al., GAUSSIAN 98, rev. A.7 (Gaussian, Inc., Pittsburgh, PA, 1998).

${ }^{20}$ T. H. Dunning, Jr. and P. J. Hay, in Modern Theoretical Chemistry, edited by H. F. Schaefer III (Plenum, New York, 1976), Vol. 3, pp. 1-28.

${ }^{21}$ P. J. Hay and W. R. Wadt, J. Chem. Phys. 82, 270 (1985).

${ }^{22}$ M. Reiher, Inorg. Chem. 41, 6928 (2002).

${ }^{23}$ See EPAPS document No. E-PRBMDO-75-067701 for UV-VIS spectra, calculated vibrational frequencies, and a movie of the shift of the Bragg peak (23 K, $1310 \mathrm{~nm}$ and $808 \mathrm{~nm}$ irradiation). For more information on EPAPS, see http://www.aip.org/ pubservs/epaps.html 
${ }^{24}$ P. Gütlich, Struct. Bonding (Berlin) 44, 83 (1981).

${ }^{25}$ K. Ichiyanagi, J. Hebert, L. Toupet, H. Cailleau, P. Guionneau, J.-F. Létard, and E. Collet, Phys. Rev. B 73, 060408(R) (2006).

${ }^{26}$ N. Huby, L. Guérin, E. Collet, L. Toupet, J. C. Ameline, H. Cailleau, T. Roisnel, T. Tayagaki, and K. Tanaka, Phys. Rev. B 69 020101(R) (2004).

${ }^{27}$ D. Chernyshov, M. Hostettler, K. Törnroos, and H. S. Bürgi, Angew. Chem., Int. Ed. 42, 3825 (2003).
${ }^{28}$ D. Chernyshov, H. B. Bürgi, M. Hostettler, and K. W. Törnroos, Phys. Rev. B 70, 094116 (2004).

${ }^{29}$ K. Nakamo, S. Kawata, K. Yoneda, A. Fuyuhiro, T. Yagi, S. Nasu, S. Morimoto, and S. Kaizaki, Chem. Commun. (Cambridge) 2004, 2892 (2004).

${ }^{30}$ M. H. Klingele, B. Moubaraki, J. D. Cashion, K. S. Murray, and S. Brooker, Chem. Commun. (Cambridge) 2005, 987 (2005). 